\title{
Low-Complexity Optimal Detection for Hybrid Space-Time Block Coding and Spatial Multiplexing
}

\author{
Yue $\mathrm{Wu}$ and Chintha Tellambura \\ Department of Electrical and Computer Engineering \\ University of Alberta \\ Edmonton, AB, Canada T6G 2V4 \\ Email: $\{$ yuewu, chintha\}@ece.ualberta.ca
}

\begin{abstract}
The combination of space-time block codes (STBC) and spatial multiplexing (SM) schemes has the advantages of diversity gain and high data rate. For such STBC/SM hybrid systems, we propose a new detector based on the block structure of STBC and the sphere decoder (SD). By employing the SD, optimal detection is performed with computational complexity much less than the brute-force maximum likelihood (ML) search. Simulation results show that the new decoder significantly outperforms the previously developed zero-forcing (ZF) group/STBC detector, the minimum mean square error (MMSE) group/STBC detector, and the MMSE and ML detectors for equivalent SM systems.
\end{abstract}

\section{INTRODUCTION}

Multiple-input and multiple-output (MIMO) systems have recently emerged as a promising technology for high daterate broadband wireless communications. This paper studies maximum likelihood (ML) detection for a hybrid MIMO system of a space-time block code (STBC) and spatial multiplexing (SM). STBCs extract diversity gain by using orthogonal designs, while SM increases the data rate and spectral efficiency by transmitting independent data streams simultaneously from multiple antennas. By combining these two schemes, both diversity gain and high data rates can be achieved simultaneously [1]-[3].

Some suboptimal detectors, namely the zero-forcing (ZF) group detector, the minimum mean square error (MMSE) group detector, and the QR group detector, have been proposed in [4] for the STBC/SM hybrid system. All these detectors incur a significant performance loss compared to the ML detector. The sphere decoder (SD) [5], [6], proposed for closest-point searches in lattices, is an efficient implementation of the ML detector. The use of the SD is further investigated in [7], [8]. In this paper, we propose a new detector combining the SD and the block structure of the STBC. Simulation results show the new detector obtains ML performance with low complexity for a wide range of SNRs.

The rest of this paper is organized as follows. In Section II, the system model is presented. Section III derives the proposed new detector. Simulation results are shown and analyzed in Section IV. Some conclusions are made in Section V.

Notation: $\mathrm{E}\{\cdot\},(\cdot)^{*},(\cdot)^{T},(\cdot)^{H}$, and $(\cdot)^{\dagger}$ denote expectation, complex conjugation, transpose, conjugate transpose, and Moore-Penrose pseudo-inverse, respectively. A complex
Gaussian variable with mean $\mu$ and variance $\sigma^{2}$ is denoted by $z \sim \mathcal{C} \mathcal{N}\left(\mu, \sigma^{2}\right)$. The $N \times N$ identity matrix is $\mathbf{I}$. The Kronecker delta is $\delta_{i, j}=1$ if $i=j$ and $\delta_{i, j}=0$ if $i \neq j$ where $i, j \in \mathbb{Z}$. An $(M, N)$ MIMO system has $M$ transmit antennas and $N$ receive antennas.

\section{SySTEM MODEL}

The STBC/SM hybrid system consists of a serial/parallel converter, a number of STBC encoders, and a detector. In this paper, we consider the Alamouti $2 \times 2$ STBC [9]. We assume the number of transmit antennas, $N_{T}$, is even. The input data stream $s_{1} s_{2} \cdots s_{N_{T}-1} s_{N_{T}}$ is split into $N_{T} / 2$ groups. Each group is sent into an independent STBC encoder. There are $N_{T} / 2$ STBC encoders, each with two transmit antennas. All the STBC encoded symbols are transmitted simultaneously. For the $i$-th STBC encoder, the input $s_{2 i} s_{2 i-1}$ is encoded and transmitted from the two antennas in two time slots. With $N_{R}$ receive antennas at the receiver, the detector provides the estimates $\hat{s}_{N_{T}} \hat{s}_{N_{T}-1} \cdots \hat{s}_{2} \hat{s}_{1}$. The system model is shown in Fig.1.

The received signal over two time slots is given by

$$
\mathbf{y}=\mathbf{H} \mathbf{s}+\mathbf{n},
$$

where $\mathbf{H}=\left[h_{l m}\right]$ is the $N_{R} \times N_{T}$ channel matrix with $h_{l m} \sim \mathcal{C N}(0,1)$ for $l=1, \cdots, N_{R}$ and $m=1, \cdots, N_{T}$ and $\mathrm{E}\left\{h_{l m} h_{l^{\prime} m^{\prime}}^{*}\right\}=\delta_{l, l^{\prime}} \delta_{m, m^{\prime}}, \mathbf{s}$ is the $N_{T} \times 2$ matrix after STBC encoding, and $\mathbf{n}$ is the $N_{R} \times 2$ matrix of additive white noise $\mathcal{C N}\left(0, \sigma_{n}^{2}\right)$ terms. We assume that $\mathbf{H}$ is constant over a block of two time slots but changes independently from block to block. The transmit signal matrix $\mathbf{s}$ can be written as

$$
\mathbf{s}=\left[\begin{array}{llll}
\mathbf{s}_{1}^{T} & \mathbf{s}_{2}^{T} & \cdots & \mathbf{s}_{N_{T} / 2}^{T}
\end{array}\right]^{T}
$$

where

$$
\mathbf{s}_{i}=\left[\begin{array}{cc}
s_{2 i-1} & s_{2 i}^{*} \\
s_{2 i} & -s_{2 i-1}^{*}
\end{array}\right], 1 \leq i \leq N_{T} / 2
$$

is the coded block from the $i$-th STBC encoder. Thus (1) can be written as

$$
\mathbf{y}=\sum_{i=1}^{N_{T} / 2} \mathbf{H}_{i} \mathbf{s}_{i}+\mathbf{n}
$$

where $\mathbf{H}=\left[\begin{array}{llll}\mathbf{H}_{1} & \mathbf{H}_{2} & \cdots & \mathbf{H}_{N_{T} / 2}\end{array}\right]$ and $\mathbf{H}_{i}$ is the virtual channel 


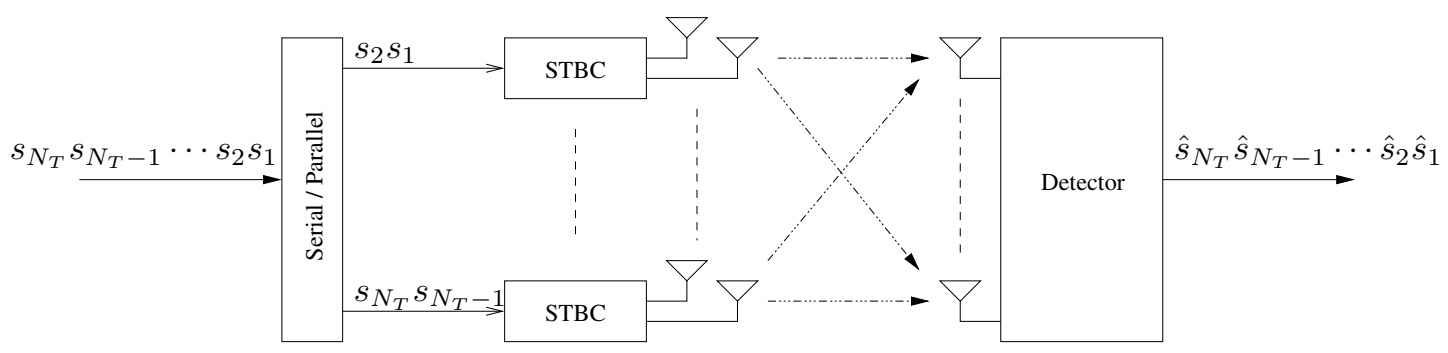

Fig. 1. System model

matrix for the $i$-th STBC encoder. It is assumed that the detector operates with the full channel state information.

\section{DETECTION OF THE STBC/SM HYBRID SYSTEM}

The detectors proposed in [4] consists of two parts. The first part is a group receiver that separates each STBC block from one another. The second part consists of a number of STBC decoders. The new detector we propose here integrates the two parts. The structure of STBC is processed before an $\mathrm{SD}$ is used to achieve maximum likelihood detection.

\section{A. MMSE group detector}

The MMSE group detector [4] is given by

$$
\begin{aligned}
\mathbf{W}_{i} & =\arg \min _{W} \mathrm{E}\left(\left\|\mathbf{W} \mathbf{y}-\mathbf{H}_{i} \mathbf{s}_{i}\right\|^{2}\right) \\
& =\mathbf{H}_{i} \mathbf{H}_{i}^{H}\left(\mathbf{H H}^{H}+\sigma_{n}^{2} \mathbf{I}_{N_{R}}\right)^{-1}, 1 \leq i \leq N_{T} / 2 .
\end{aligned}
$$

With the corresponding virtual channel matrix $\mathbf{H}_{i}$, the output of the MMSE group detector is processed by an STBC decoder to obtain the estimation of $s_{2 i-1}$ and $s_{2 i}$.

\section{B. ZF group detector}

ZF group detectors for $(3,3)$ and $(4,4)$ systems are proposed in [10]. Here we propose a ZF group detector for an $(8,8)$ system.

The $8 \times 8$ channel matrix decomposed into four $4 \times 4$ matrices is given by

$$
\mathbf{H}=\left[\begin{array}{ll}
\mathbf{A}_{4} & \mathbf{B}_{4} \\
\mathbf{C}_{4} & \mathbf{D}_{4}
\end{array}\right]
$$

where $\mathbf{A}_{4}, \mathbf{B}_{4}, \mathbf{C}_{4}$, and $\mathbf{D}_{4}$ are all $4 \times 4$ matrices. The $\mathrm{ZF}$ group detector at this level is

$$
\mathbf{W}_{4}=\left[\begin{array}{ll}
\mathbf{B}_{4}^{-1} & -\mathbf{D}_{4}^{-1} \\
\mathbf{A}_{4}^{-1} & -\mathbf{C}_{4}^{-1}
\end{array}\right] .
$$

Thus

$$
\mathbf{W}_{4} \mathbf{H}=\left[\begin{array}{cc}
\mathbf{B}_{4}^{-1} \mathbf{A}_{4}-\mathbf{D}_{4}^{-1} \mathbf{C}_{4} & \mathbf{0}_{4} \\
\mathbf{0}_{4} & \mathbf{A}_{4}^{-1} \mathbf{B}_{4}-\mathbf{C}_{4}^{-1} \mathbf{D}_{4}
\end{array}\right]
$$

and the output of this detector is $\tilde{\mathbf{y}}=\mathbf{W}_{4} \mathbf{H s}$, which can be written as

$$
\begin{aligned}
& {\left[\begin{array}{c}
\tilde{\mathbf{y}}_{1} \\
\tilde{\mathbf{y}}_{2}
\end{array}\right]=\left(\mathbf{B}_{4}^{-1} \mathbf{A}_{4}-\mathbf{D}_{4}^{-1} \mathbf{C}_{4}\right)\left[\begin{array}{l}
\mathbf{s}_{1} \\
\mathbf{s}_{2}
\end{array}\right]} \\
& {\left[\begin{array}{c}
\tilde{\mathbf{y}}_{3} \\
\tilde{\mathbf{y}}_{4}
\end{array}\right]=\left(\mathbf{A}_{4}^{-1} \mathbf{B}_{4}-\mathbf{C}_{4}^{-1} \mathbf{D}_{4}\right)\left[\begin{array}{c}
\mathbf{s}_{3} \\
\mathbf{s}_{4}
\end{array}\right]}
\end{aligned}
$$

For (9), the equivalent channel matrix $\mathbf{H}_{e q}$ can be written as

$$
\mathbf{H}_{e q}=\left(\mathbf{B}_{4}^{-1} \mathbf{A}_{4}-\mathbf{D}_{4}^{-1} \mathbf{C}_{4}\right)=\left[\begin{array}{ll}
\mathbf{A}_{2} & \mathbf{B}_{2} \\
\mathbf{C}_{2} & \mathbf{D}_{2}
\end{array}\right],
$$

where $\mathbf{A}_{2}, \mathbf{B}_{2}, \mathbf{C}_{2}$, and $\mathbf{D}_{2}$ are all $2 \times 2$ matrices. The $\mathrm{ZF}$ group detector at this level is

$$
\mathbf{W}_{2}=\left[\begin{array}{ll}
\mathbf{B}_{2}^{-1} & -\mathbf{D}_{2}^{-1} \\
\mathbf{A}_{2}^{-1} & -\mathbf{C}_{2}^{-1}
\end{array}\right] .
$$

Thus the detection in [10] for a $(4,4)$ system can be followed directly to get the estimation of $s_{1}, \cdots, s_{4}$. The detection for $s_{5}, \cdots, s_{8}$ is similar. This method can also be extended to any system with the number of antennas being $2^{k}, k=2,3, \cdots$.

\section{New detector}

Unlike the previous detectors that separate the group detector and STBC decoder, our new detector combines the block structure of STBC and the SD. The basic idea of SD is the following: Instead of searching all the signal points, search is limited to only those signal points that lie in a certain hypersphere of radius $r$ around a given point, which reduces the search space and required computations [11].

Since the $\mathbf{s}$ in (1) is an $N_{T} \times 2$ matrix, the SD can not be employed directly with (1). In fact, (1) can be written as

$$
\begin{aligned}
& {\left[\begin{array}{cc}
y_{11} & y_{12} \\
\vdots & \vdots \\
y_{N_{R}, 1} & y_{N_{R}, 2}
\end{array}\right]=} \\
& {\left[\begin{array}{ccc}
h_{11} & \cdots & h_{1, N_{T}} \\
\vdots & \ddots & \vdots \\
h_{N_{R}, 1} & \cdots & h_{N_{R}, N_{T}}
\end{array}\right]\left[\begin{array}{cc}
s_{1} & -s_{2}^{*} \\
s_{2} & s_{1}^{*} \\
\vdots & \vdots \\
s_{N_{T}-1} & -s_{N_{T}}^{*} \\
s_{N_{T}} & s_{N_{T}-1}^{*}
\end{array}\right]+\mathbf{n} .}
\end{aligned}
$$

We may transform (13) as

$$
\mathbf{y}^{\prime}=\mathbf{H}^{\prime} \mathbf{s}^{\prime}+\mathbf{n}^{\prime}
$$

where

$$
\mathbf{y}^{\prime}=\left[\begin{array}{lllll}
y_{11} & y_{12}^{*} & \cdots & y_{N_{R}, 1} & y_{N_{R}, 2}^{*}
\end{array}\right]^{T},
$$


$\mathbf{H}^{\prime}=\left[\begin{array}{ccccc}h_{11} & h_{12} & \cdots & h_{1, N_{T}-1} & h_{1, N_{T}} \\ h_{12}^{*} & -h_{11}^{*} & \cdots & h_{1, N_{T}}^{*} & -h_{1, N_{T}-1}^{*} \\ \vdots & \vdots & \ddots & \vdots & \vdots \\ h_{N_{R}, 1} & h_{N_{R}, 2} & \cdots & h_{N_{R}, N_{T}-1} & h_{N_{R}, N_{T}} \\ h_{N_{R}, 2}^{*} & -h_{N_{R}, 1}^{*} & \cdots & h_{N_{R}, N_{T}}^{*} & -h_{N_{R}, N_{T}-1}^{*}\end{array}\right]$,

and

$$
\mathbf{s}^{\prime}=\left[\begin{array}{lllll}
s_{1} & s_{2} & \cdots & s_{N_{T}-1} & s_{N_{T}}
\end{array}\right]^{T} .
$$

Thus $\mathbf{s}^{\prime}$, the part that contains $\mathbf{s}$, has become a vector in (14), which means the SD can now be applied to obtain the ML estimation of $\mathbf{s}^{\prime}$.

The maximum likelihood detector for (14) is given by

$$
\tilde{\mathbf{s}}^{\prime}=\arg \min _{\mathbf{s}^{\prime} \in \mathcal{Q}^{N_{T}}}\left\|\mathbf{y}^{\prime}-\mathbf{H}^{\prime} \mathbf{s}^{\prime}\right\|^{2} .
$$

We consider quadrature amplitude modulation (QAM) for $\mathbf{s}^{\prime}$. Since a QAM constellation can be decoupled into two real pulse amplitude modulation (PAM) constellations, solving (18) is equivalent to solving

$$
\tilde{\mathbf{s}}^{\prime \prime}=\arg \min _{\mathbf{s}^{\prime \prime} \in \mathcal{Q}^{2 N_{T}}}\left\|\mathbf{y}^{\prime \prime}-\mathbf{H}^{\prime \prime} \mathbf{s}^{\prime \prime}\right\|^{2},
$$

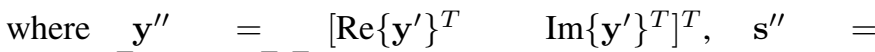
$\left[\operatorname{Re}\left\{\mathbf{s}^{\prime}\right\}^{T} \operatorname{Im}\left\{\mathbf{s}^{\prime}\right\}^{T}\right]^{T}$, and

$$
\mathbf{H}^{\prime \prime}=\left[\begin{array}{cc}
\operatorname{Re}\left\{\mathbf{H}^{\prime}\right\} & -\operatorname{Im}\left\{\mathbf{H}^{\prime}\right\} \\
\operatorname{Im}\left\{\mathbf{H}^{\prime}\right\} & \operatorname{Re}\left\{\mathbf{H}^{\prime}\right\}
\end{array}\right] .
$$

Eq. (19) can be written as

$$
\begin{aligned}
\tilde{\mathbf{s}}^{\prime \prime} & =\arg \min _{\mathbf{s}^{\prime \prime} \in \mathcal{Q}^{2 N_{T}}}\left(\mathbf{H}^{\prime \prime \dagger} \mathbf{y}^{\prime \prime}-\mathbf{s}^{\prime \prime}\right)^{H} \mathbf{R}^{H} \mathbf{R}\left(\mathbf{H}^{\prime \prime} \mathbf{y}^{\prime \prime}-\mathbf{s}^{\prime \prime}\right) \\
& =\arg \min _{\mathbf{s}^{\prime \prime} \in \mathcal{Q}^{2 N_{T}}}\left(\mathbf{s}^{\prime \prime}-\hat{\mathbf{s}}^{\prime \prime}\right)^{H} \mathbf{R}^{H} \mathbf{R}\left(\mathbf{s}^{\prime \prime}-\hat{\mathbf{s}}^{\prime \prime}\right),
\end{aligned}
$$

where $\hat{\mathbf{s}}^{\prime \prime}=\mathbf{H}^{\prime \prime} \mathbf{y}^{\prime \prime}$ and $\mathbf{R}$ is an upper triangular matrix such that $\mathbf{R}^{H} \mathbf{R}=\mathbf{H}^{\prime \prime}{ }^{H} \mathbf{H}^{\prime \prime}$. Let the entries of $\mathbf{R}$ be denoted by $r_{i j}$, $i \leq j$. In this paper we assume $N_{R} \geq N_{T}$ and the diagonal terms of $\mathbf{R}$ are non-zero $\left(r_{i i} \neq 0\right)$. A candidate is inside the hypersphere of radius $r$ when

$$
\left(\mathbf{s}^{\prime \prime}-\hat{\mathbf{s}}^{\prime \prime}\right)^{H} \mathbf{R}^{H} \mathbf{R}\left(\mathbf{s}^{\prime \prime}-\hat{\mathbf{s}}^{\prime \prime}\right)=\sum_{i=1}^{2 N_{T}} r_{i i}^{2}\left\|s_{i}^{\prime \prime}-\rho_{i}\right\|^{2} \leq r^{2},
$$

where $\rho_{i}=\hat{s}_{i}^{\prime \prime}-\sum_{j=i+1}^{2 N_{T}} \frac{r_{i j}}{r_{i i}}\left(s_{j}^{\prime \prime}-\hat{s}_{j}^{\prime \prime}\right)$. The initial radius may be chosen according to the noise variance [6]. The initial radius can be increased if it is too small to contain any candidate solution. Since each term in the above equation is nonnegative, a necessary condition for $\mathbf{s}^{\prime \prime}$ to lie inside the hypersphere is that $r_{2 N_{T}, 2 N_{T}}^{2}\left\|s_{2 N_{T}}^{\prime \prime}-\hat{s}_{2 N_{T}}^{\prime \prime}\right\|^{2} \leq r^{2}$ or equivalently

$$
\left\lceil\hat{s}_{2 N_{T}}^{\prime \prime}-\frac{r}{r_{2 N_{T}, 2 N_{T}}}\right\rceil \leq s_{2 N_{T}}^{\prime \prime} \leq\left\lfloor\hat{s}_{2 N_{T}}^{\prime \prime}+\frac{r}{r_{2 N_{T}, 2 N_{T}}}\right\rfloor \text {, }
$$

where $\lceil\cdot\rceil$ denotes the smallest integer greater than or equal to its argument. $\lfloor\cdot\rfloor$ denotes the largest integer less than or equal to its argument. The same process continues for $s_{2 N_{T}-1}$ and so on until it reaches $s_{1}$, obtaining a vector point satisfying (21). The radius $r$ is then updated and the search space is limited by the new radius. The above process continues until no further points are found within the hypersphere. This process yields the ML solution.

\section{Simulation Results}

Fig. 2 (4QAM) and Fig. 3 (16QAM) show the error performance of an uncoded $(4,4)$ STBC/SM hybrid system with two STBC encoders at the transmit side. The performance of a $(2,4)$ SM system (no STBC encoders) is included, and it has the same date throughput as the $(4,4)$ STBC/SM system. For 4QAM, the ZF group/STBC detector performs the worst among all detectors. The MMSE group/STBC outperforms the $\mathrm{ZF}$ group/STBC by less than $2 \mathrm{~dB}$ for most of the SNR range. The new detector outperforms the MMSE group/STBC by more than $5 \mathrm{~dB}$ at a bit error rate (BER) of $10^{-3}$. The MMSE detector for the equivalent SM system performs slightly better than the ZF group/STBC detector. The ML detector for the SM system performs worse than the MMSE/STBC detector in low SNR but outperforms the latter in high SNR. For 16QAM, the performance of the ZF group/STBC detector and the MMSE detector for the SM system is very close. The MMSE group/STBC detector has a $2 \mathrm{~dB}$ gain over both of them in low SNR. In high SNR, the performance of the ML detector for the SM system has a more than $1 \mathrm{~dB}$ gain over the three. The new detector significantly outperforms the other four by more than $4 \mathrm{~dB}$ at the BER of $10^{-2}$.

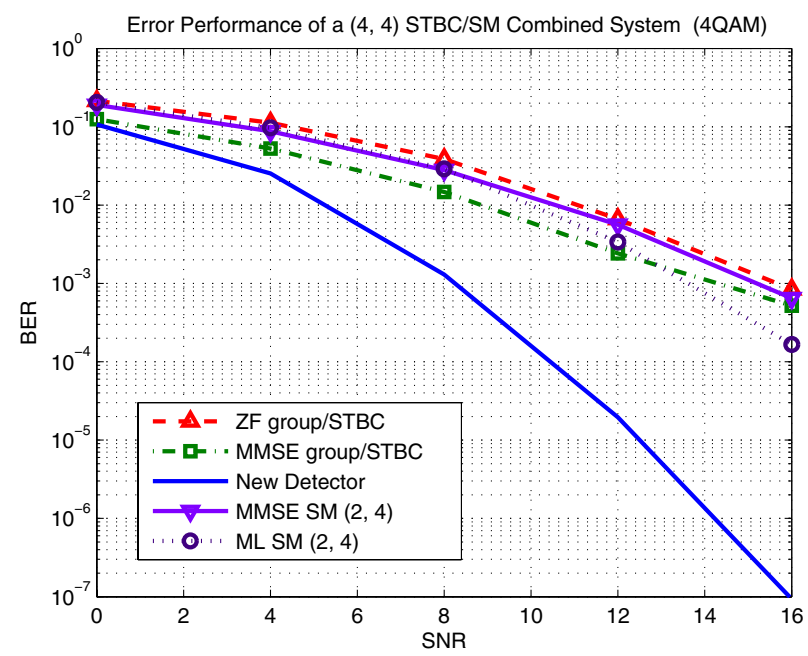

Fig. 2. Error Performance of a $(4,4)$ STBC/SM system (4QAM)

Fig. 4 shows the performance of the detectors in an uncoded $(8,8) \mathrm{STBC} / \mathrm{SM}$ hybrid system. There are 4 STBC encoders at the transmit side. 4QAM is employed. The $(4,8)$ SM system has equivalent data throughput to the $(8,8) \mathrm{STBC} / \mathrm{SM}$ system. In high SNR, the MMSE group/STBC detector gains $6 \mathrm{~dB}$ over the ZF group/STBC, which has the worst performance among all. The MMSE detector for the SM system outperforms the MMSE group/STBC detector by $3 \mathrm{~dB}$ in high SNR. The ML detector for the SM system has a $3 \mathrm{~dB}$ gain over the MMSE detector for the same SM system. The new detector 


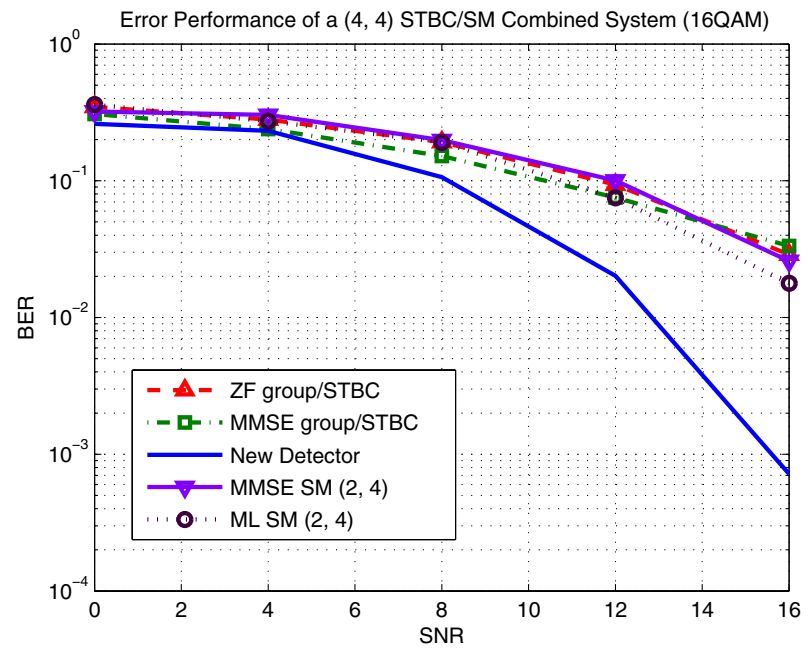

Fig. 3. Error Performance of a $(4,4)$ STBC/SM system (16QAM)

outperforms the ML detector for the SM system by $4 \mathrm{~dB}$ and has the best performance among all.

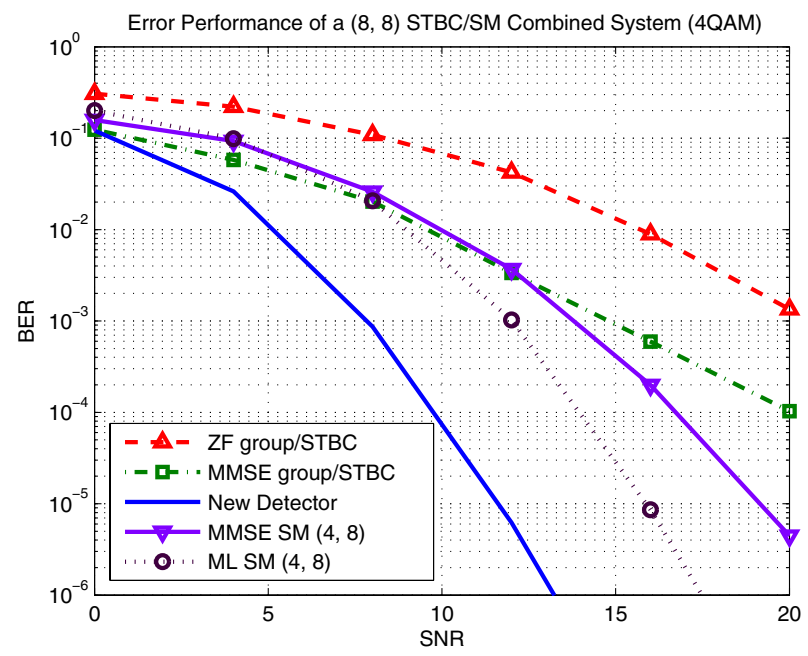

Fig. 4. Error Performance of a $(8,8)$ STBC/SM system (4QAM)

Based on empirical simulations, Fig. 5 shows the computational complexity of a $(4,4)$ STBC/SM hybrid system. All curves are plotted for 4QAM. In low SNR, the new detector has a high computational complexity, but the complexity decreases significantly as the SNR increases. In high SNR, the complexity of the new detector is close to that of the MMSE group/STBC detector, which has a higher complexity than the ZF group/STBC detector. The complexity of both the detectors for the $(2,4)$ SM system is low as a result of the small number of transmit antennas. The complexity of the naive ML detector for the equivalent SM system is expected to increase exponentially with the increase of the number of transmit antennas.

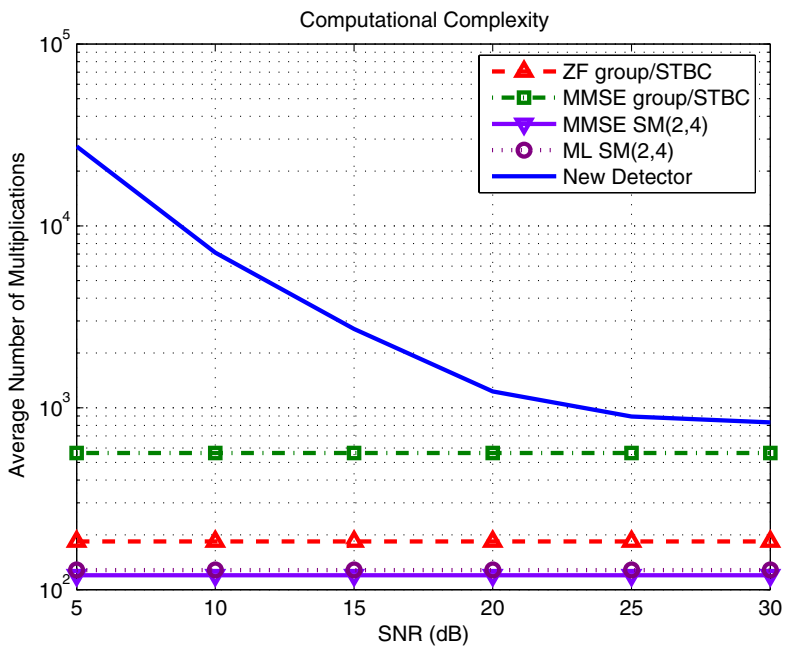

Fig. 5. Computational complexity of a $(4,4)$ STBC/SM system (4QAM)

\section{CONClusion}

In this paper, we have proposed a new maximum likelihood detector for the STBC/SM hybrid system. The new detector combines the block structure of the STBC encoder and an SD. The computational complexity of the new detector is much less than the brute-force ML search. Simulation results show that the new detector significantly outperforms the ZF group/STBC detector, the MMSE/STBC group detector, and the MMSE and ML detectors for equivalent SM systems.

\section{REFERENCES}

[1] L. Zheng and D. Tse, "Diversity and multiplexing: a fundamental tradeoff in multiple antenna channels," IEEE Trans. Inform. Theory, vol. 49, no. 5, pp. 1073-1096, May 2003.

[2] R. Heath and A. Paulraj, "Switching between diversity and multiplexing in MIMO systems," IEEE Trans. Commun., vol. 53, no. 6, pp. 962-968, Jun 2005.

[3] A. L. F. de Almeida, J. C. M. Mota, W. C. Freitas Jr., F. R. P. Cavalcanti, "Performance of MIMO systems with hybrid of transmit diversity and spatial multiplexing," Proceedings of Brazilian Telecommunications Symposium (SBT'03), Oct 2003.

[4] L. Zhao and V. K. Dubey, "Detection schemes for space-time block code and spatial multiplexing combined system," IEEE Communications Letters, vol. 9, no. 1, pp. 49-51, Jan 2005.

[5] U. Fincke and M. Pohst, "Improved methods for calculating vectors of short length in a lattice, including a complexity analysis," Mathematics of Computation, vol. 44, pp. 463-471, Apr 1985.

[6] M. O. Damen, A. Chkeif and J. C. Belfiore, "Lattice codes decoder for space-time codes," IEEE Commun. Letters, vol. 4, pp. 161-163, May 2000.

[7] T. Cui and C. Tellambura, "An efficient generalized sphere decoder for rank-deficient MIMO systems," IEEE Communication Letters, vol. 9, no. 5, pp. 423-425, May 2005.

[8] — - "Approximate ML detection for MIMO systems using multistage sphere decoding," IEEE Signal Processing Letters, vol. 12, no. 3, pp. 222-225, Mar 2005.

[9] S. M. Alamouti, "A simple transmitter diversity scheme for wireless communication," IEEE J. Select. Areas. Commun., vol. 16, pp. 14511458, Oct. 1998.

[10] L. Zhao and V. K. Dubey, "Transmit diversity and combining scheme for spatial multiplexing over correlated channels," Proc. Vehicular Technology conference, vol. 1, pp. 380-383, May 2004.

[11] M. O. Damen, H. El Gamal and G. Caire, "On maximum-likelihood detection and the search for the closest lattice point," IEEE Trans. Inform. Theory, vol. 49, no. 10, pp. 2389-2402, Oct 2003. 